EPJ Web of Conferences 108, 02015 (2016)

DOI: $10.1051 /$ epjconf/201610802015

(C) Owned by the authors, published by EDP Sciences, 2016

\title{
Relativistic Three-Nucleon Calculations within the Bethe-Salpeter Approach
}

\author{
S.G. Bondarenko ${ }^{1, a}$, V.V. Burov ${ }^{1, b}$, and S.A. Yurev ${ }^{1,2, c}$ \\ ${ }^{1}$ Joint Institute for Nuclear Research, Dubna, 141980, Russia \\ ${ }^{2}$ Far Eastern Federal University, Vladivostok, 690950, Russia
}

\begin{abstract}
The relativistic properties of the three-nucleon system are investigated using the Faddeev equations within the Bethe-Salpeter approach. The nucleon-nucleon interaction is chosen in a separable form. The Gauss quadrature method is used to calculate the integrals. The system of the integral equations is solved by an iterative method. The binding energy and the partial-wave amplitudes $\left({ }^{1} S_{0}\right.$ and $\left.{ }^{3} S_{1}\right)$ of the triton are found.
\end{abstract}

\section{Introduction}

Three-body calculations in nuclear physics are of great interest for describing three-nucleon bound states $\left({ }^{3} \mathrm{He}, \mathrm{T}\right)$, processes of elastic, inelastic and deep inelastic scattering the leptons off light nuclei and also the hadron-deuteron reactions (for example, $p d \rightarrow p d, p d \rightarrow p p n$ ). The study of the nuclei ${ }^{3} \mathrm{He}$ and $\mathrm{T}$ is also interesting because it allows us to investigate further (in addition to the case of the deuteron) the evolution of the bound nucleon thereby contributing to the explanation of so-called EMC-effect. In quantum mechanics the Faddeev equations are commonly used to describe threeparticle systems. The main feature of Faddeev equations is that all particles interact through a pair potential.

At high momentum transfers relativistic effects should be taken into account. The Bethe-Salpeter (BS) [1] equation is one of the most consistent approaches to describe the NN interaction. In this formalism, one deals with systems of nontrivial integral equations for both the NN scattered states and the bound state - the deuteron. To solve a system of integral equations, it is convenient to use a separability Ansatz [2] for the interaction kernel in the BS equation. In this case, one can transform the integral equations into systems of algebraic linear ones which are easy to solve. The parameters of the interaction kernel are found from the analysis of the phase shifts and inelasticity, low-energy parameters and deuteron properties (binding energy, moments, etc.).

The relativistic three-particle systems are described by Faddeev equations within the BS approach, the so called Bethe-Salpeter-Faddeev equations. In this paper, the three-nucleon system is studied with the simplest Yamaguchi separable potential [3]. All nucleons have equal masses and scalar propagators are used instead of spinor ones for simplicity. The spin-isospin structure of the nucleons

\footnotetext{
a e-mail: bondarenko@jinr.ru

be-mail: burov@theor.jinr.ru

ce-mail: yurev@jinr.ru
}

This is an Open Access article distributed under the terms of the Creative Commons Attribution License 4.0, which permits unrestricted use, distribution, and reproduction in any medium, provided the original work is properly cited. 
is taken into account by using the so-called recoupling-coefficient matrix. The work mainly follows the ideas of the article [4].

The paper is organized as follows. In Sec. 2, the two-particle problem is considered, in Sec. 3, three-particle equations. In Sec. 4 calculations and results are given. A summary is done in Sec. 5.

\section{Two particle case}

Since the formalism of the Faddeev equations is based on the properties of the nucleon-nucleon pair interaction only, the discussion starts with preliminaries of the two-body problem.

The Bethe-Salpeter equation for the relativistic two-particle system is:

$$
T\left(p, p^{\prime} ; s\right)=V\left(p, p^{\prime}\right)+\frac{i}{4 \pi^{3}} \int d^{4} k V(p, k) G(k ; s) T\left(k, p^{\prime} ; s\right),
$$

where $T\left(p, p^{\prime} ; s\right)$ is the two-particle $T$-matrix and $V\left(p, p^{\prime}\right)$ is the kernel (potential) of the nucleonnucleon interaction. The free two-particle Green function $G(k ; s)$ is expressed, for simplicity, through the scalar propagator of the nucleons

$$
G^{-1}(k ; s)=\left[(P / 2+k)^{2}-m_{N}^{2}+i \epsilon\right]\left[(P / 2-k)^{2}-m_{N}^{2}+i \epsilon\right] .
$$

To solve (1), the separability Ansatz for the nucleon-nucleon potential $V\left(p, p^{\prime}\right)$ is used (rank-one)

$$
V\left(p_{0}, p, p_{0}^{\prime}, p^{\prime}\right)=\lambda g\left(p_{0}, p\right) g\left(p_{0}^{\prime}, p^{\prime}\right)
$$

In this case the two-particle $T$-matrix gets the simple form:

$$
T\left(p_{0}, p, p_{0}^{\prime}, p^{\prime} ; s\right)=\tau(s) g\left(p_{0}, p\right) g\left(p_{0}^{\prime}, p^{\prime}\right)
$$

where

$$
[\tau(s)]^{-1}=\lambda^{-1}-\frac{i}{4 \pi^{3}} \int_{-\infty}^{\infty} d k^{0} \int_{0}^{\infty} k^{2} d k g^{2}\left(k^{0}, k\right) G\left(k^{0}, k ; s\right) .
$$

The simplest relativistic Yamaguchi-type form factor $g_{Y}\left(p_{0}, p\right)$ is used

$$
g_{Y}\left(p_{0}, p\right)=\frac{1}{-p_{0}^{2}+p^{2}+\beta^{2}}
$$

with parameters $\beta$ and $\lambda$ chosen such as to describe experimental data (deuteron binding energy, lowenergy scattering parameter and phase shifts). The values of the parameters are given in Table 1.

Table 1. Parameters for $\tau(s)$ and $g_{Y}\left(p_{0}, p\right)$ for S-states

\begin{tabular}{ccc}
\hline Parameter & ${ }^{1} S_{0}$ & ${ }^{3} S_{1}$ \\
\hline$\lambda\left(\mathrm{GeV}^{4}\right)$ & -1.12087 & -3.15480 \\
$\beta(\mathrm{GeV})$ & 0.287614 & 0.279731 \\
\hline
\end{tabular}




\section{Three-particle case}

The relativistic three-particle system can be described by the Bethe-Salpeter-Faddeev equations

$$
\left[\begin{array}{l}
T^{(1)} \\
T^{(2)} \\
T^{(3)}
\end{array}\right]=\left[\begin{array}{l}
T_{1} \\
T_{2} \\
T_{3}
\end{array}\right]-\left[\begin{array}{ccc}
0 & T_{1} G_{1} & T_{1} G_{1} \\
T_{2} G_{2} & 0 & T_{2} G_{2} \\
T_{3} G_{3} & T_{3} G_{3} & 0
\end{array}\right]\left[\begin{array}{c}
T^{(1)} \\
T^{(2)} \\
T^{(3)}
\end{array}\right],
$$

where the full matrix $T=\sum_{i=1}^{3} T^{(i)}, G_{i}$ is the two-particle Green function below, ( $i j n$ stand for cyclic permutations of $(1,2,3))$ :

$$
G_{i}\left(k_{j}, k_{n}\right)=1 /\left(k_{j}^{2}-m_{N}^{2}+i \epsilon\right) /\left(k_{n}^{2}-m_{N}^{2}+i \epsilon\right),
$$

and $T_{i}$ denote the two-particle $T$-matrix,

$$
T_{i}\left(k_{1}, k_{2}, k_{3} ; k_{1}^{\prime}, k_{2}^{\prime}, k_{3}^{\prime}\right)=(2 \pi)^{4} \delta^{(4)}\left(K_{i}-K_{i}^{\prime}\right) T_{i}\left(k_{j}, k_{n} ; k_{j}^{\prime}, k_{n}^{\prime}\right) .
$$

For the system of equal-mass particles the Jacobi momenta can be written as

$$
p_{i}=\frac{1}{2}\left(k_{j}-k_{n}\right), q_{i}=\frac{1}{3} K-k_{i}, K=k_{1}+k_{2}+k_{3} .
$$

Using the expressions (10) the equation (7) results in:

$$
\begin{aligned}
& T^{(i)}\left(p_{i}, q_{i} ; p_{i}^{\prime}, q_{i}^{\prime} ; s\right)=(2 \pi)^{4} \delta^{(4)}\left(q_{i}-q_{i}^{\prime}\right) T_{i}\left(p_{i} ; p_{i}^{\prime} ; s\right) \\
& -i \int \frac{d p_{i}^{\prime \prime}}{(2 \pi)^{4}} T_{i}\left(p_{i} ; p_{i}^{\prime \prime} ; s\right) G_{i}\left(k_{j}^{\prime \prime}, k_{n}^{\prime \prime}\right)\left[T^{(j)}\left(p_{j}^{\prime \prime}, q_{i}^{\prime \prime} ; p_{i}^{\prime}, q_{i}^{\prime} ; s\right)+T^{(n)}\left(p_{i}^{\prime \prime}, q_{i}^{\prime \prime} ; p_{i}^{\prime}, q_{i}^{\prime} ; s\right)\right] .
\end{aligned}
$$

It is suitable to introduce the amplitude $\Psi^{(i)}\left(p_{i}, q_{i} ; s\right)$ for the bound state of the three particles,

$$
\Psi^{(i)}\left(p_{i}, q_{i} ; s\right)=\left\langle p_{i}, q_{i}\left|T^{(i)}\right| M_{B}\right\rangle \equiv \Psi_{L M}(p, q ; s),
$$

where $M_{B}=\sqrt{s}=3 m_{N}-E_{B}$ is the mass of the bound state (triton) and $s=K^{2}$ is the squared total momentum. For the equal-mass case all $\Psi^{(i)}$ functions are equal to each other.

The total orbital angular momenta of the triton can be presented as $\boldsymbol{L}=\boldsymbol{l}+\boldsymbol{\lambda}$, where $\boldsymbol{l}$ is the angular momentum corresponding to the nucleon pair with the relative impulse $\boldsymbol{p}$ and $\lambda$ is the angular momentum corresponding to the relative impulse $\boldsymbol{q}$.

The angular dependence of the amplitude is separated by means of the factorization

$$
\Psi_{L M}(p, q ; s)=\sum_{a \lambda} \Psi_{\lambda L}^{(a)}\left(p_{0},|\mathbf{p}|, q_{0},|\mathbf{q}| ; s\right) \mathcal{Y}_{\lambda L M}^{(a)}(\hat{\mathbf{p}}, \hat{\mathbf{q}})
$$

with the angular part

$$
\boldsymbol{Y}_{\lambda L M}^{(a)}(\hat{\mathbf{p}}, \hat{\mathbf{q}})=\sum_{m \mu} C_{l m \lambda \mu}^{L M} Y_{l m}(\hat{\mathbf{p}}) Y_{\lambda \mu}(\hat{\mathbf{q}}),
$$

where the two-nucleon state with spin $s$, angular moment $l$ and total momentum $j\left(a \equiv{ }^{2 s+1} l_{j}\right)$ is introduced, $C$ is the Clebsch-Gordan coefficients and $Y$ are spherical harmonics.

For the rank-one separable two-nucleon interaction the amplitude $\Psi_{\lambda L}^{(a)}$ can be written as

$$
\Psi_{\lambda L}^{(a)}\left(p_{0}, p, q_{0}, q ; s\right)=g^{(a)}\left(p_{0}, p\right) \tau^{(a)}(s) \Phi_{\lambda L}^{(a)}\left(q_{0}, q ; s\right),
$$


where the function $\Phi_{\lambda L}^{(a)}$ satisfies the integral equation

$$
\begin{array}{r}
\Phi_{\lambda L}^{(a)}\left(q_{0}, q ; s\right)=\frac{i}{4 \pi^{3}} \sum_{a^{\prime} \lambda^{\prime}} \int_{-\infty}^{\infty} d q_{0}^{\prime} \int_{0}^{\infty} q^{\prime 2} d q^{\prime} Z_{\lambda \lambda^{\prime}}^{\left(a a^{\prime}\right)}\left(q_{0}, q ; q_{0}^{\prime}, q^{\prime} ; s\right) \\
\frac{\tau^{\left(a^{\prime}\right)}\left[\left(\frac{2}{3} \sqrt{s}+q_{0}^{\prime}\right)^{2}-q^{\prime 2}\right]}{\left(\frac{1}{3} \sqrt{s}-q_{0}^{\prime}\right)^{2}-q^{\prime 2}-m^{2}+i \epsilon} \Phi_{\lambda^{\prime}}^{\left(a^{\prime}\right)}\left(q_{0}^{\prime}, q^{\prime} ; s\right),
\end{array}
$$

with

$$
\begin{array}{r}
Z_{\lambda \lambda^{\prime}}^{\left(a a^{\prime}\right)}\left(q_{0}, q ; q_{0}^{\prime}, q^{\prime} ; s\right)=C_{\left(a a^{\prime}\right)} \int d\left(\cos \vartheta_{q q^{\prime}}\right) K_{\lambda \lambda^{\prime} L}^{\left(a a^{\prime}\right)}\left(q, q^{\prime}, \cos \vartheta_{q q^{\prime}}\right) \\
\frac{g^{(a)}\left(-\frac{1}{2} q_{0}-q_{0}^{\prime},\left|\mathbf{q} / 2+\mathbf{q}^{\prime}\right|\right) g^{\left(a^{\prime}\right)}\left(q_{0}+\frac{1}{2} q_{0}^{\prime},\left|\mathbf{q}+\mathbf{q}^{\prime} / 2\right|\right)}{\left(\frac{1}{3} \sqrt{s}+q_{0}+q_{0}^{\prime}\right)^{2}-\left(\mathbf{q}+\mathbf{q}^{\prime}\right)^{2}-m_{N}^{2}+i \epsilon}
\end{array}
$$

and

$$
\begin{array}{r}
K_{\lambda \lambda^{\prime} L}^{\left(a a^{\prime}\right)}\left(q, q^{\prime}, \cos \vartheta_{q q^{\prime}}\right)=(4 \pi)^{3 / 2} \frac{\sqrt{2 \lambda+1}}{2 L+1} \\
\sum_{m m^{\prime}} C_{l m \lambda 0}^{L m} C_{l^{\prime} m^{\prime} \lambda^{\prime} m-m^{\prime}}^{L m} Y^{*}{ }_{l m}(\vartheta, 0) Y_{l^{\prime} m^{\prime}}\left(\vartheta^{\prime}, 0\right), Y_{\lambda^{\prime} m-m^{\prime}}\left(\vartheta_{q q^{\prime}}, 0\right)
\end{array}
$$

where

$$
\cos \vartheta=\frac{\frac{q}{2}+q^{\prime} \cos \vartheta_{q q^{\prime}}}{\left|\frac{\mathbf{q}}{2}+\mathbf{q}^{\prime}\right|}, \quad \cos \vartheta^{\prime}=\frac{q+\frac{q^{\prime}}{2} \cos \vartheta_{q q^{\prime}}}{\left|\mathbf{q}+\frac{\mathbf{q}^{\prime}}{2}\right|}
$$

and $C_{\left(a a^{\prime}\right)}$ is the spin-isospin recoupling-coefficient matrix.

Considering the ground state of the three-particle state $L=0$ and assuming the internal orbital angular momenta equal to zero, $l=\lambda=0$, one should take into into account only two states: $a=$ $\left({ }^{1} S_{0},{ }^{3} S_{1}\right)$. In this case one gets $K_{000}^{00}=1$ and the system of integral equations reads

$$
\begin{array}{r}
\Phi^{(a)}\left(q_{0}, q ; s\right)=\frac{i}{4 \pi^{3}} \sum_{a^{\prime}} \int d q_{0}^{\prime} \int q^{\prime 2} d q^{\prime} Z^{\left(a a^{\prime}\right)}\left(q_{0}, q, q_{0}^{\prime}, q^{\prime} ; s\right) \\
\\
\frac{\tau^{\left(a^{\prime}\right)}\left[\left(\frac{2}{3} \sqrt{s}+q_{0}^{\prime}\right)^{2}-q^{\prime 2}\right]}{\left(\frac{1}{3} \sqrt{s}-q_{0}^{\prime}\right)^{2}-q^{\prime 2}-m_{N}^{2}+i \epsilon} \Phi^{\left(a^{\prime}\right)}\left(q_{0}^{\prime}, q^{\prime} ; s\right) .
\end{array}
$$

Here the so-called effective energy-dependent potential $Z$ is

$$
\begin{array}{r}
Z^{\left(a a^{\prime}\right)}\left(q_{0}, q, q_{0}^{\prime}, q^{\prime} ; s\right)=C_{\left(a a^{\prime}\right)} \int_{-1}^{1} d\left(\cos \vartheta_{q q^{\prime}}\right) \\
\frac{g^{(a)}\left(-\frac{1}{2} q_{0}-q_{0}^{\prime},\left|-\frac{1}{2} \mathbf{q}-\mathbf{q}^{\prime}\right|\right) g^{\left(a^{\prime}\right)}\left(q_{0}+\frac{1}{2} q_{0}^{\prime},\left|\mathbf{q}+\frac{1}{2} \mathbf{q}^{\prime}\right|\right)}{\left(\frac{1}{3} \sqrt{s}+q_{0}+q_{0}^{\prime}\right)^{2}-\left(\mathbf{q}+\mathbf{q}^{\prime}\right)^{2}-m_{N}^{2}+i \epsilon}
\end{array}
$$

with

$$
C_{\left(a a^{\prime}\right)}=\left[\begin{array}{cc}
\frac{1}{4} & -\frac{3}{4} \\
-\frac{3}{4} & \frac{1}{4}
\end{array}\right] .
$$

The system of integral equations (19)-(20) has a number of singularities, however in the case of the bound three-particle system $\left(\sqrt{s}<3 m_{N}\right)$ all these singularities do not cross the path of integration on $q_{0}$ and thus do not affect the Wick-rotation procedure $q_{0} \rightarrow i q_{4}$.

The system (19)-(20) got after the Wick-rotation procedure is analytically well defined and can be solved by different numerical methods. One of them is discussed in the next section. 


\section{Solution and results}

In order to solve the system of integral equations the method of iterations is used. The mappings of the variables of integration $q[0, \infty)$ and $q_{4}(-\infty, \infty)$ to $[-1,1]$ are defined.

The binding energy of the three-nucleon system satisfies the following condition (for details, see [5]):

$$
\left.\lim _{n \rightarrow \infty} \frac{\Phi_{n}(s)}{\Phi_{n-1}(s)}\right|_{s=M_{B}^{2}}=1,
$$

where $n$ is number of iteration.

The calculations result in the binding energy $E_{B}=10.99 \mathrm{MeV}$ which is to be compared to the experimental value $8.48 \mathrm{MeV}$. The difference can originate in the simplifying assumption that a separable kernel (rank-one) of the nucleon-nucleon interaction can be used.

The obtained partial-wave amplitudes are shown in figures 1 to 3 .


Figure 1. Real (left) and imaginary (right) parts of the ${ }^{1} S_{0}$ partial-wave state versus $q_{4}$ at different $q$ values: solid line - at $q=0 \mathrm{Fm}^{-1}$, dashed line - at $q=0.5 \mathrm{Fm}^{-1}$ and dotted-dashed line - at $q=1 \mathrm{Fm}^{-1}$
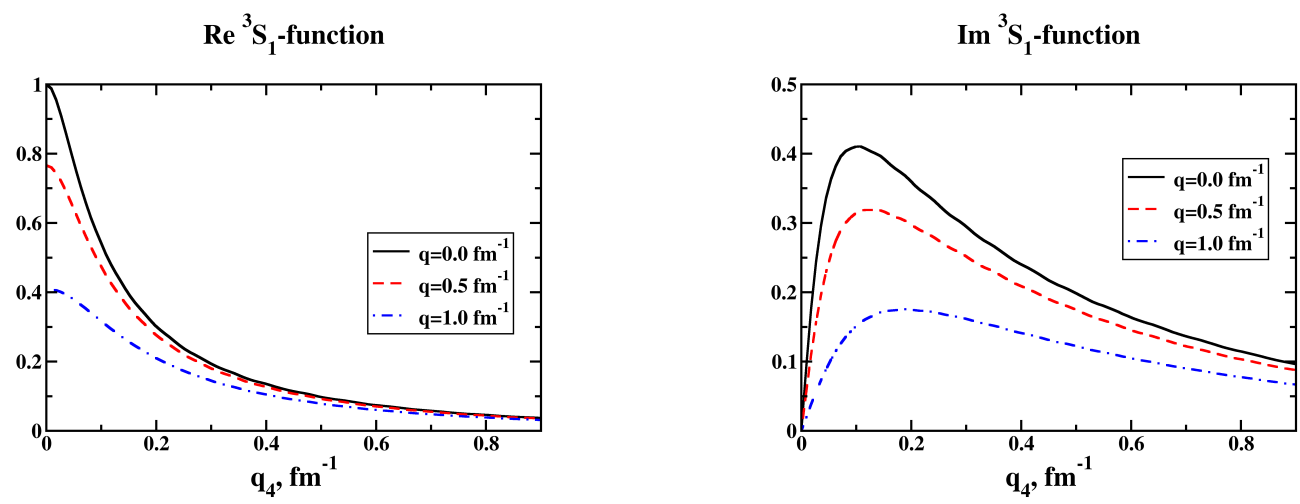

Figure 2. Real (left) and imaginary (right) parts of the ${ }^{3} S_{1}$ partial-wave state versus $q_{4}$ at different $q$ values: solid line - at $q=0 \mathrm{Fm}^{-1}$, dashed line - at $q=0.5 \mathrm{Fm}^{-1}$ and dotted-dashed line - at $q=1 \mathrm{Fm}^{-1}$ 

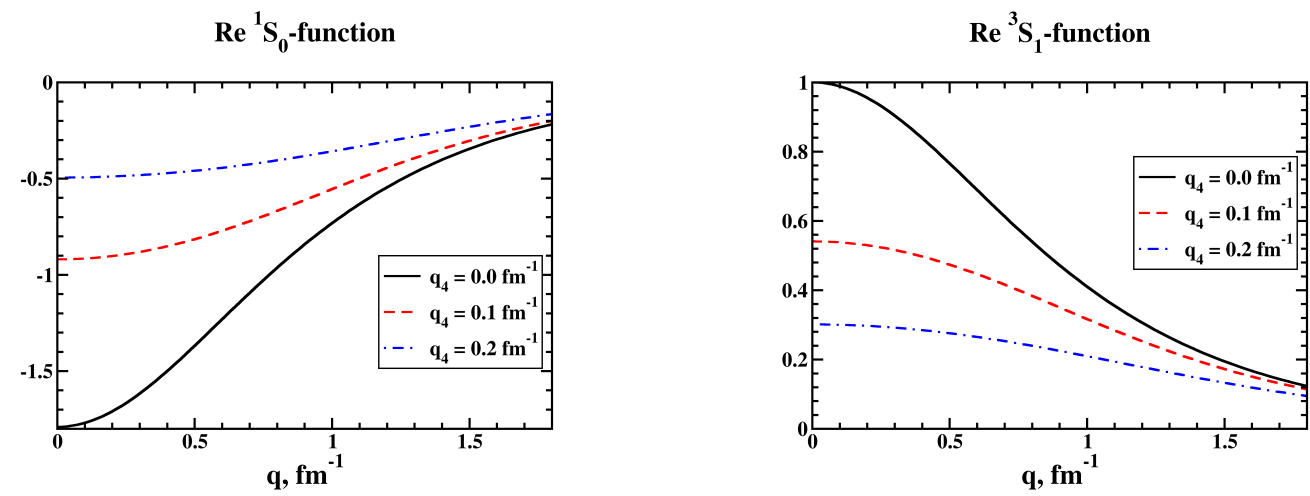

Figure 3. Real parts of the ${ }^{1} S_{0}$ (left) and ${ }^{3} S_{1}$ (right) partial-wave state versus $q$ at different $q_{4}$ values: solid line -at $q_{4}=0 \mathrm{Fm}^{-1}$, dashed line - at $q_{4}=0.1 \mathrm{Fm}^{-1}$ and dotted-dashed line - at $q_{4}=0.2 \mathrm{Fm}^{-1}$

\section{Summary}

In the paper the three-body system is investigated using Bethe-Salpeter-Faddeev (BSF) equations. The nucleon-nucleon interaction is taken in the rank-one separable form. The relativistic generalization of Yamaguchi-type functions are chosen in the calculations. The parameters of the nucleon-nucleon potential for the ${ }^{1} S_{0}$ and ${ }^{3} S_{1}$ partial-wave states reproduce the low-energy scattering parameter and deuteron properties as well as phase shifts up to the laboratory energy 100-120 MeV. The BSF integral equations are solved by using iterations method. The binding energy of the triton and the amplitudes of the ${ }^{1} S_{0}$ and ${ }^{3} S_{1}$ partial-wave states of the triton are found.

A large overestimation of the binding energy of the triton is found. To improve the theoretical results, the rank of the separable kernel should be increased. Other partial-wave states, such as $P$ - and $D$-states, and spinor propagators for nucleons should be taken into account as well.

\section{Acknowledgments}

One of the authors (S.G. Bondarenko) thanks the organizers of MMCP 2015 and personally Professor Michal Hnatič for invitation, support and hospitality.

\section{References}

[1] E.E. Salpeter, H.A. Bethe, Phys. Rev. 84, 1232 (1951)

[2] S.G. Bondarenko, V.V. Burov, A.V. Molochkov, G.I. Smirnov, H. Toki, Prog. Part. Nucl. Phys. 48, 449 (2002)

[3] Y. Yamaguchi, Phys. Rev. 95, 1628 (1954); Y. Yamaguchi, Y. Yamaguchi, Phys. Rev. 95, 1635 (1954)

[4] G. Rupp and J. A. Tjon, Phys. Rev. C 37, 1729 (1988)

[5] R. A. Malfliet and J. A. Tjon, Nucl. Phys. A127,161-168 (1969) 